ARTICLE

Received 1 Jun 2014 | Accepted 10 Nov 2014 | Published 18 Dec 2014

DOI: $10.1038 /$ ncomms6815

\title{
Epigenetic regulation of Atrophin1 by lysine-specific demethylase 1 is required for cortical progenitor maintenance
}

Feng Zhang ${ }^{1,2}$, Dan $X u^{1,2}$, Ling Yuan ${ }^{1,2}$, Yiming Sun ${ }^{1} \&$ Zhiheng $X u^{1,3}$

Lysine-specific demethylase 1 (LSD1) is involved in gene regulation and development; however, its precise function, molecular targets and underlying mechanisms during development are poorly understood. Here we show that LSD1 is required for neuronal progenitor cell (NPC) maintenance during cortical development. A ChIP-seq analysis identified a LSD1-binding site (LBAL) downstream of Atrophin1 (ATN1). Surprisingly, tranylcypromine (LSD1 inhibitor) treatment increased H3K4 methylation at LBAL, leading to ATN1 repression and NPC differentiation. Knockdown of LSD1 and ATN1 phenocopied each other in inducing NPC premature differentiation and depletion, which could be rescued by ATN1 overexpression, suggesting that LSD1 controls NPC differentiation via regulation of ATN1 methylation status and expression. The involvement of LSD1 in ATN1 expression and NPC maintenance were confirmed in knockout mice. These findings hint at the potential application for the clinical drug, tranylcypromine, in the prevention and/or treatment of ATN1-associated degenerative disease, dentatorubral-pallidoluysian atrophy.

\footnotetext{
${ }^{1}$ State Key Laboratory of Molecular Developmental Biology, Institute of Genetics and Developmental Biology, Chinese Academy of Sciences, 1 West Beichen Road, Beijing 100101, China. ${ }^{2}$ University of Chinese Academy of Sciences, Beijing 100101, China. ${ }^{3}$ Parkinson's Disease Center, Beijing Institute for Brain Disorders, Beijing 100101, China. Correspondence and requests for materials should be addressed to Z.X. (email: zhxu@genetics.ac.cn).
} 
pigenetic regulation of gene expression plays a critical role in many biological processes, including cell reprogramming, development, tumorigenesis and ageing ${ }^{1-5}$. Histone methylation is one type of epigenetic modification which, when perturbed, leads to various diseases ${ }^{6-8}$.

The development of the mammalian neocortex requires a balance between self-renewal and differentiation of neural progenitor/precursor cells (NPCs, predominantly radial glial cells) followed by neuronal migration and maturation ${ }^{9-12}$. Lysine-specific demethylase 1 (LSD1), a histone demethylase for mono- or dimethylated H3K4 and H3K9 (ref. 13), has been indicated in different aspects of brain development, including pituitary development ${ }^{14}$, cortical neuron migration ${ }^{15}$ and adult NPC proliferation ${ }^{16}$. LSD1 is also required for embryonic stem cell differentiation ${ }^{17}$. In addition, LSD1 was recently identified as a target of the microRNA miR-137; overexpression of miR-137 induced premature differentiation and migration of immature neurons ${ }^{18}$. Thus, although this implicates that LSD1 may be involved in coordinating NPC proliferation versus differentiation, detailed information on its molecular targets and mechanisms of action is still lacking.

Dentatorubral-pallidoluysian atrophy (DRPLA) is an autosomal dominant neuronal degenerative disease characterized by ataxia, choreoathetosis and cognitive dysfunction, including dementia, resulting from polyglutamine (polyQ) expansion in Atrophin1 (ATN1) ${ }^{99-21}$. ATN1 interacts with, and modulates the activity of, the transcriptional repressor $\mathrm{TLX}^{22}$. ATN1 knockout mice are viable ${ }^{23}$, but the function of ATN1 in brain development has not yet been examined.

The present study investigated the role of LSD1 in the neocortex to determine whether epigenetic changes in gene expression during embryonic development affect progenitor cell maintenance. We provided evidence here that ANT1 is a direct target of LSD1 and that knockdown or knockout of LSD1 leads to the downregulation of ATN1 expression. Knockdown of either LSD1 or ATN1 induces significant premature differentiation and depletion of NPCs, and it can be rescued by ATN1 expression. Moreover, the clinical LSD1 inhibitor, tranylcypromine, suppressed ATN1 expression, suggesting that it could be applied to the treatment of adult-onset DRPLA.

\section{Results}

LSD1 depletion results in premature NPC differentiation. The expression of LSD1 in the developing rat brain was first examined. LSD1 immunoreactivity was observed in the nucleus in all layers of the cortex, including the proliferative ventricular and subventricular zones (VZ and SVZ, respectively), the intermediate zone (IZ) through which newly born neurons migrate and the nascent cortical plate (CP) (Fig. 1a and Supplementary Fig. 1a). Two short hairpin RNA (shRNA)/DsRed constructs were found to be highly efficient in knocking down both overexpressed and endogenous LSD1 (Figs 1b,c and 3g, and Supplementary Fig. 6a). These shRNA constructs were electroporated into rat cortex via in utero electroporation (IUE) at embryonic day 16.5 (E16.5) and the brains were examined 4 days later (E20.5). In control embryos transfected with a control shRNA/DsRed construct, DsRed ${ }^{+}$cells were distributed in all the layers (Fig. 1d). In contrast, on LSD1 knockdown most of the transfected cells were found in the IZ, with very few in the $\mathrm{CP}$, consistent with a previous report that LSD1 depletion inhibits neuronal migration ${ }^{15}$. However, much fewer cells were observed in the VZ and SVZ compared with controls (Fig. 1d). Cortical neurons arise on a chronological schedule with subtypes of neurons emerging in a defined order ${ }^{24,25}$; therefore, we performed the IUE experiment from E15.5 to E18.5 similar to what we did previously ${ }^{26}$. Similar results of NPC depletion were obtained (Fig. 1e), indicating that LSD1 may regulate NPC development at different stages.

We examined whether the loss of NPC induced by LSD1 knockdown is due to increased cell death, by staining the brain sections with the activated form of caspase 3, and found no apparent increase of cell death (Supplementary Fig. 1b). In addition, co-transfection of a shRNA-resistant LSD1 construct rescued the depletion of NPCs in the VZ and SVZ (Supplementary Fig. 2a-c), thereby excluding the possibility of shRNA off-target effects.

To determine whether the depletion of transfected cells in the proliferative zones corresponded to cell fate changes, cells transfected with LSD1 shRNA/DsRed from E15.5 to 18.5 were examined for the expression of the progenitor markers Sox 2 and Tbr2-which label apical progenitors/radial glial cells of the VZ and intermediate/basal progenitors of the SVZ, respectively-as well as the early neuronal marker Tuj1. LSD1 knockdown resulted in substantial decrease of Sox $2^{+}$and $\mathrm{Tbr} 2^{+}$cells, and a corresponding increase of Tuj1 ${ }^{+}$cells, and these could be rescued by co-expression of human LSD1 (Fig. 2a and Supplementary Fig. 2d). These results indicate that the loss of LSD1 in NPCs induces their premature differentiation into neurons.

To determine whether the observed effect was due to the demethylase activity of LSD1, cortical progenitor cells dissociated at E16.5 were treated with the LSD1 inhibitor tranylcypromine ${ }^{27}$ and examined $24 \mathrm{~h}$ later by staining cells with the progenitor cell marker Nestin and the differentiation marker Tuj1. Inhibition of LSD1 activity induced the differentiation of NPCs, as indicated by the significantly increased number of Tuj ${ }^{+}$cells and concomitant decrease in Nestin ${ }^{+}$cells (Fig. 2b).

To further confirm the role of LSD1 in neuronal differentiation, LSD1 $1^{\text {flox/flox }}$ mice were crossed with Nestin-Cre mice to generate the LSD1 brain-specific conditional knockout (LSD1 cKO) mice. Loss of LSD1 expression in cKO mouse brains was verified by immunostaining and western blotting (Fig. $3 \mathrm{~h}$ and Supplementary Fig. 3a). cKO mice have smaller brains and thinner cortices compared with their wild-type littermates (Supplementary Fig. 3a). E15.5 wild-type or LSD1 cKO mice were then injected with 5-bromodeoxyuridine (BrdU) and analysed $24 \mathrm{~h}$ later. Similar to that of LSD1 knockdown, BrdU ${ }^{+}$ cells in the VZ were depleted in the LSD1 cKO brains (Fig. 2c), with most $\mathrm{BrdU}^{+}$cells localized in the SVZ and much less cells reaching the IZ, in contrast to their wild-type littermates (Supplementary Fig. 3b). More importantly, the fraction of $\mathrm{Ki}^{-} / \mathrm{BrdU}^{+}$cells increased significantly in the LSD1 cKO brains, indicating that more $L S D 1 \mathrm{cKO}$ cells underwent cell cycle exit and premature differentiation (Fig. 2c,d). Probably due to the premature differentiation, the number of cells incorporated with BrdU decreased substantially in LSD1 cKO mice (Supplementary Fig. 3c). We also dissected NPCs from E14.5 mice, cultured for $48 \mathrm{~h}$ and examined the Nestin and Tuj1 markers. Similar to LSD1 inhibitor treatment, knockout of LSD1 induced differentiation of NPCs, as revealed by the reduction of Nestin ${ }^{+}$cells and increase of Tuj $1^{+}$cells (Supplementary Fig. 3d,e). Taken together, our results demonstrate that LSD1 is required for the maintenance of NPCs during cortical development.

LSD1 positively regulates ATN1 expression. To further investigate the mechanism by which LSD1 maintains the progenitor pool, chromatin immunoprecipitation sequencing (ChIP-seq) was performed in E16.5 brain homogenates to identify potential targets of LSD1. Genes encoding histones were the most highly represented among the potential targets (Supplementary Table 1), consistent with previous reports and the known function of LSD1 (ref. 28). A novel candidate gene was ATN1. Interestingly, the 
a

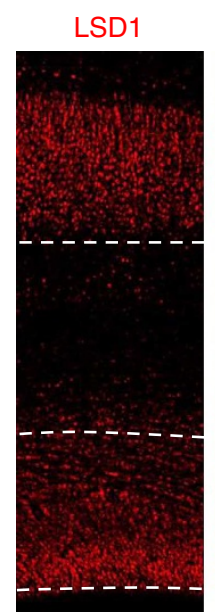

d
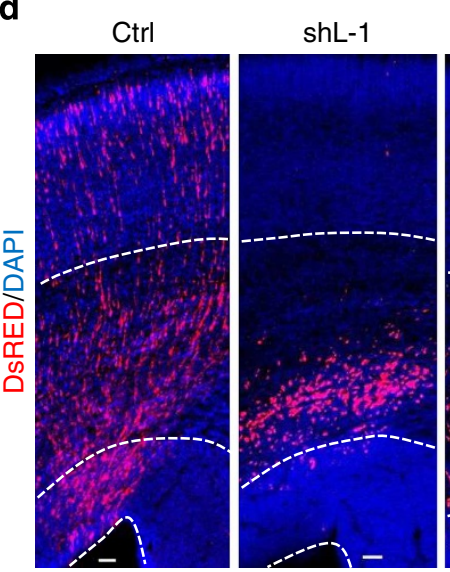

Merge

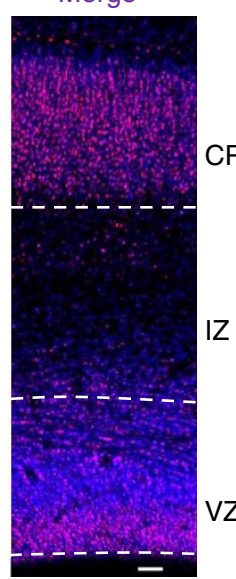

shL-2

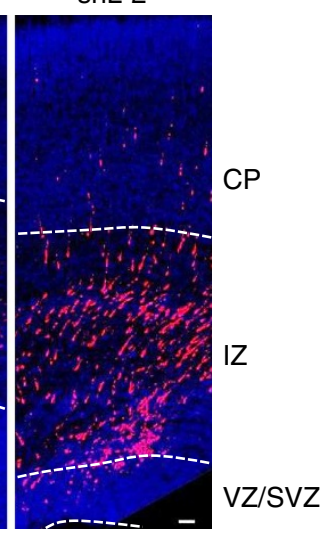

VZ/SVZ b

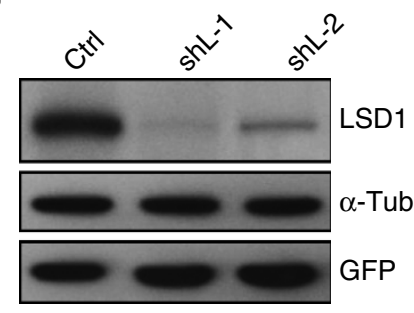

C

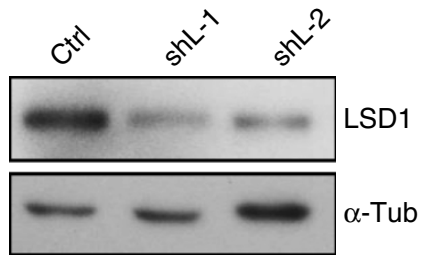

e

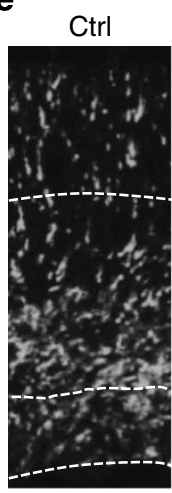
shL-1
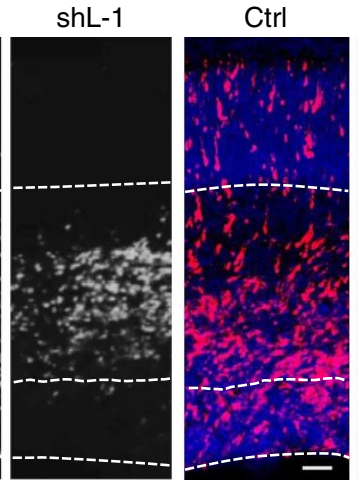

shL-1
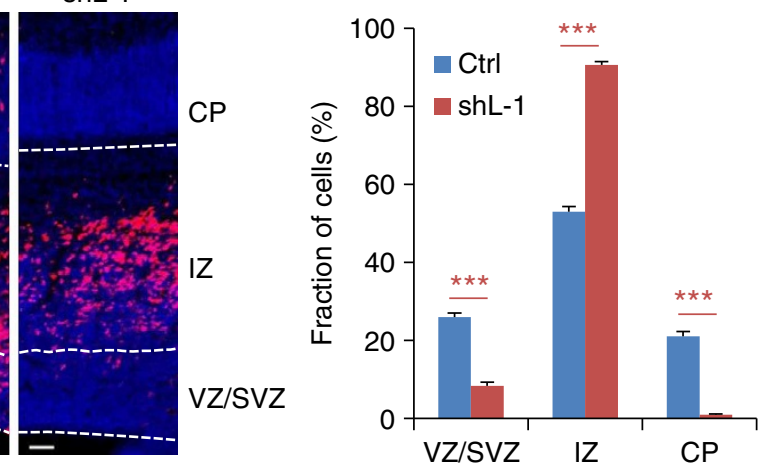

Figure 1 | LSD1 knockdown leads to altered cell distribution in the cortex. (a) Coronal sections from E18.5 rat cortex stained with 4',6-diamidino-2phenylindole (DAPI) to label nuclei (blue) and an antibody against LSD1 (red). (b,c) Endogenous and overexpressed LSD1 are efficiently knocked down by LSD1 shRNA (shL). (b) pEGFP-N1-LSD1 was co-transfected with a control shRNA (shCtrl) or shL into HEK293 cells; $24 \mathrm{~h}$ later, cell lysates were analysed by immunoblotting with anti-LSD1 antibody, with $\alpha$-tubulin serving as a loading control and GFP as a control for transfection efficiency. (c) Ctrl or shL was transfected into N2A cells; $48 \mathrm{~h}$ later, DsRed ${ }^{+}$cells were collected by fluorescence-activated cell sorting and analysed by immunoblotting with anti-LSD1 antibody. (d) Coronal sections of rat brains electroporated in utero with Ctrl or shL at E16.5 and examined at E20.5. Right panel: quantification of DsRed ${ }^{+}$ cell distribution in the cortex. Data represent mean \pm s.e.m. ${ }^{\star \star \star} P<0.001$ (Ctrl, $n=26 / 8$; shL-1, $n=24 / 7$; shL-2, $n=10 / 3$; one-way analysis of variance). (e) Cortex electroporated at E15.5 and analysed at E18.5. Data represent mean \pm s.e.m. ${ }^{\star \star \star} P<0.001$ (Ctrl, $n=21 / 7$; shL-1, $n=27 / 9$; Student's $t$-test). $n$, number of slices from different brains. Scale bars, $50 \mu \mathrm{m}$.

analysis revealed a putative LSD1-binding site downstream of the ATN1 coding region, as opposed to within the promoter (Fig. 3a and Supplementary Fig. 4). A homology search indicated that this sequence, which was named LBAL (LSD1-binding site at the ATN1 locus), is conserved in rodents and humans (Fig. $3 \mathrm{~b}$ and
Supplementary Fig. 5). Intriguingly, ChIP-seq results by a previous report indicate that the binding site of the SET1/MLL histone methyltransferase complex subunit, Dpy-30, overlaps with LBAL (Fig. 3a) ${ }^{29}$. The binding of LSD1 and K4-dimethylated histone H3 (dimethyl H3K4) to LBAL was confirmed by ChIP- 
a
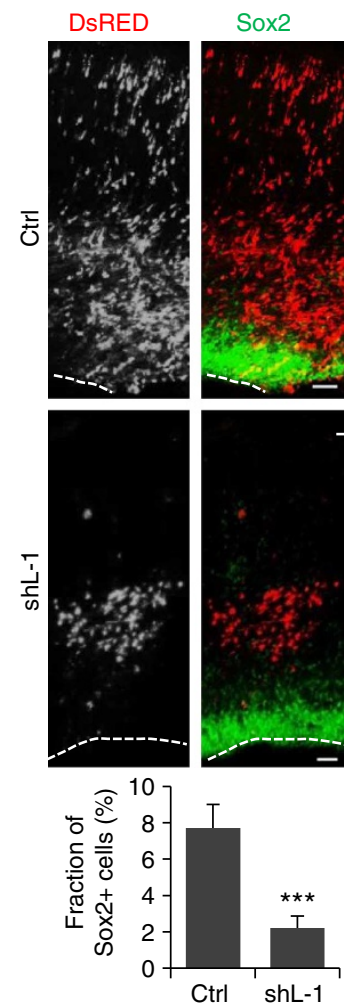

b
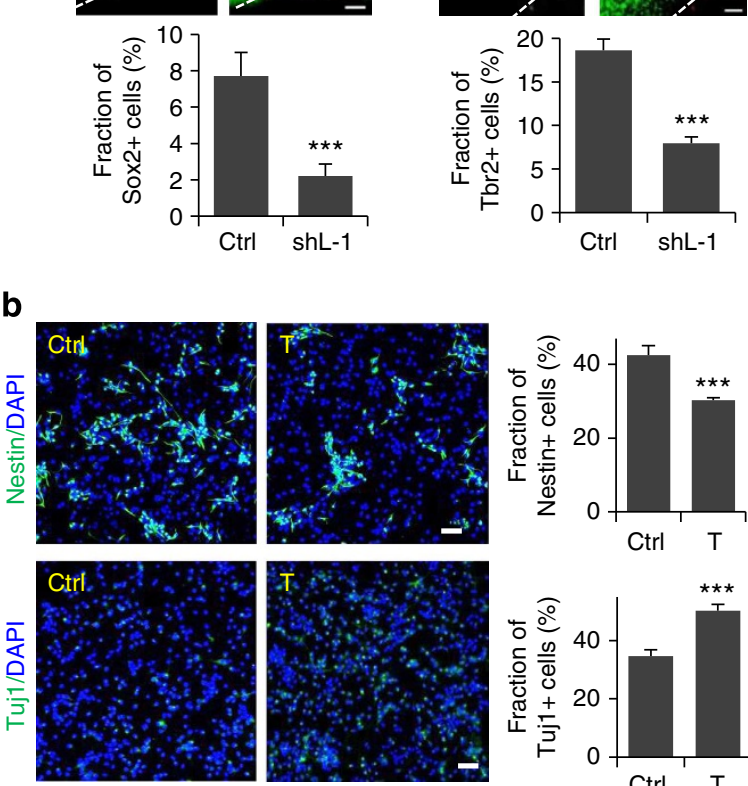

Ctrl shL-1
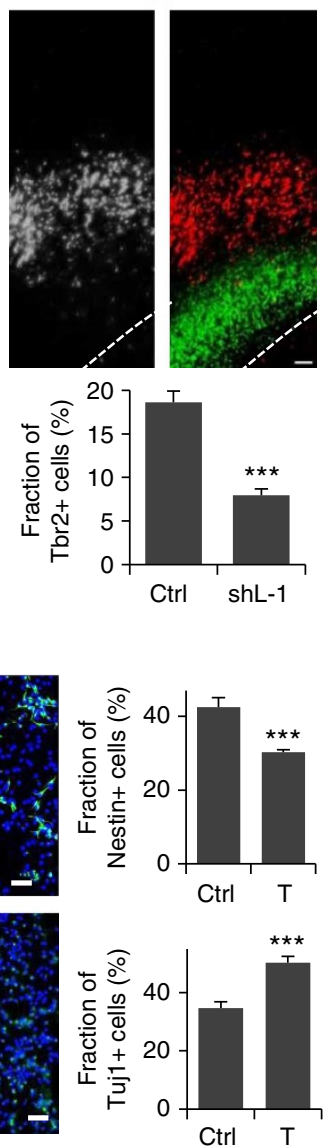

c
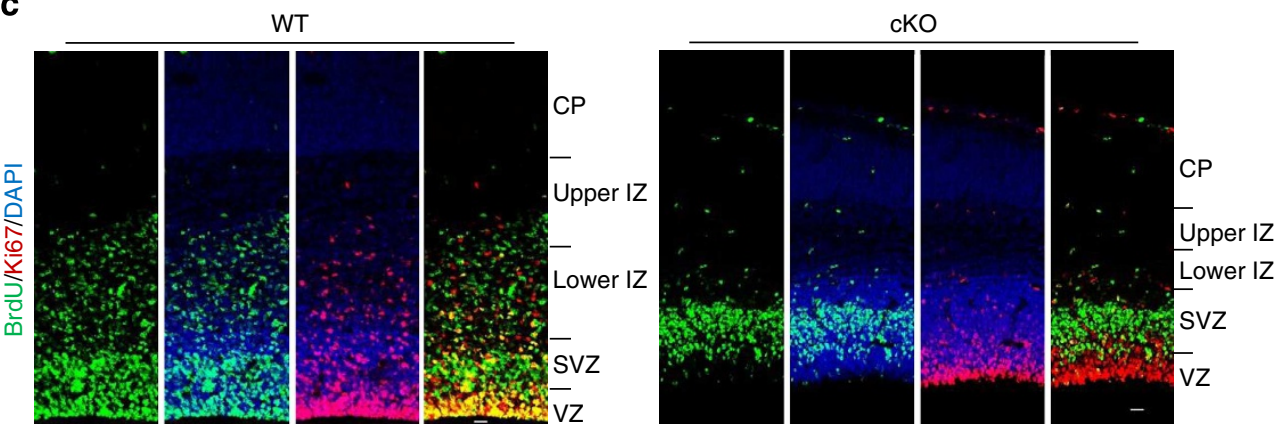

Figure 2 | LSD1 depletion leads to premature differentiation of NPCs. (a) LSD1 knockdown leads to premature differentiation of NPCs. Coronal sections of cortices electroporated with shCtrl or shL-1 at E15.5 were examined at E18.5 by staining with antibodies against Sox2, Tbr2, or Tuj1; the quantitative analysis is shown in the lower panels. Data represent mean \pm s.e.m. ${ }^{\star \star \star} P<0.001$, $t$ - test. Sox2 (Ctrl, $n=7 / 7 ;$ shL-1, $\left.n=10 / 10\right) ;$ Tbr2 (Ctrl, $n=7 / 7 ;$ shL-1, $\left.n=10 / 10\right) ;$ Tuj1 (Ctrl, $n=7 / 7$; shL-1, $n=8 / 8$ ). $n$, number of slices from different brains. (b) E16.5 rat cortices were dissociated and cultured in DMEM/B27/bFGF/EGF for $24 \mathrm{~h}$, then treated with saline ( $\mathrm{Ctrl})$ or tranylcypromine $(\mathrm{T}, 10 \mu \mathrm{M})$ for $24 \mathrm{~h}$. After fixation, cells were stained for Nestin and Tuj1. Quantitative analyses are shown in the lower panels. Data represent mean \pm s.e.m. ${ }^{\star \star \star} P<0.001$ (Student's t-test, $>4,000$ cells were counted for each group). (c,d) Coronal cortical sections from E16.5 wild-type or brain-specific LSD1-cKO mice injected with BrdU (100 $\mu \mathrm{g} \mathrm{kg}^{-1}$ ) at E15.5 were immunostained with BrdU and Ki67 antibodies (c). Nuclei were labelled with 4',6-diamidino-2-phenylindole (DAPI). Scale bar, $20 \mu \mathrm{m}$. The fractions of BrdU ${ }^{+} / \mathrm{Ki}^{-} 7^{-}$cells were quantified in d. Data represent means \pm s.e.m. ${ }^{\star \star \star} P<0.001$ (WT: $n=9 / 3$; cKO: $n=9 / 3$; Student's $t$-test). $n$, number of slices from different brains. 
a

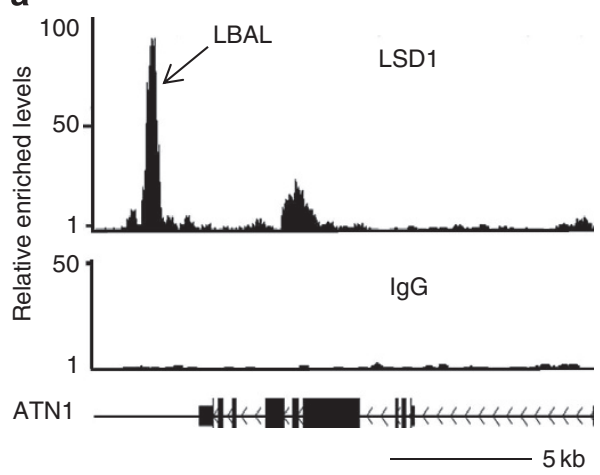

b

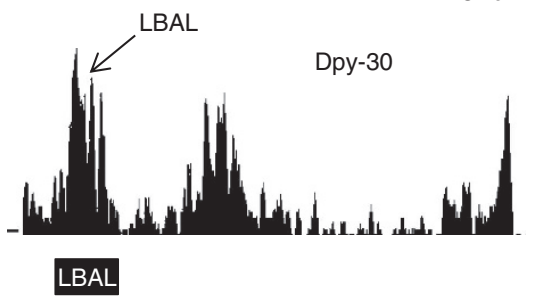

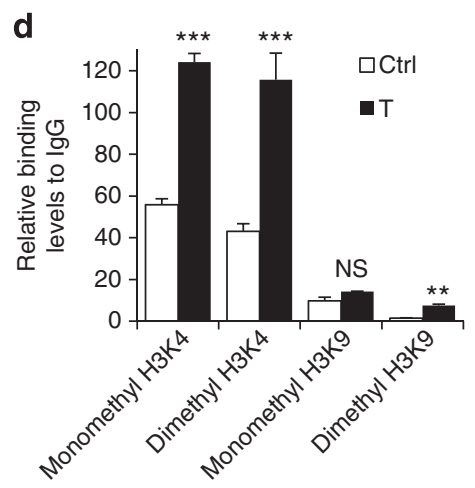

g

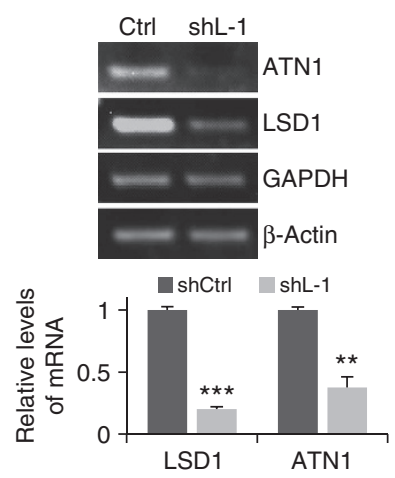

e

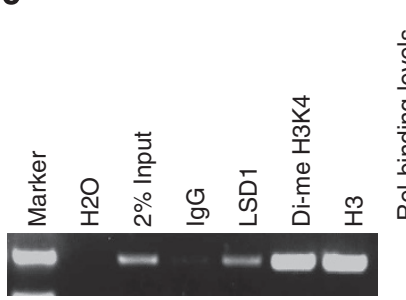

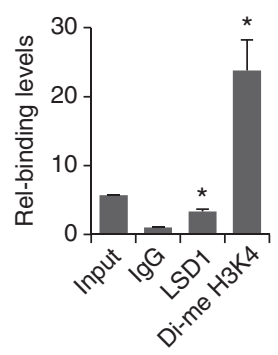

f
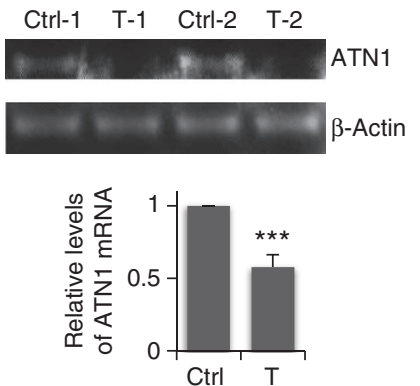

h

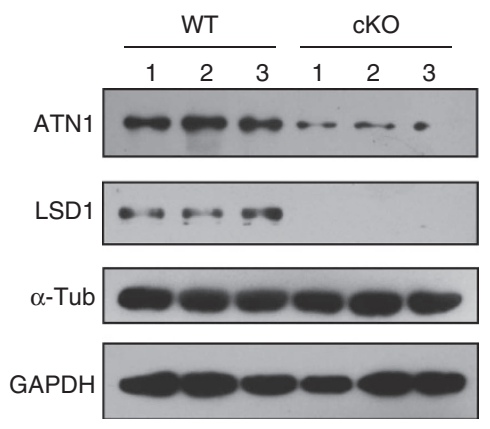

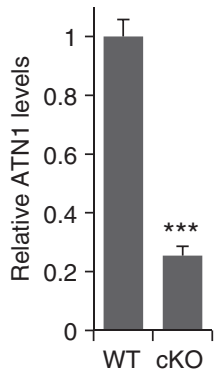

Figure 3 | LSD1 binds LBAL and regulates dimethyl H3K4 level and ATN1 expression. (a) LSD1 binds to the LBAL site downstream of ATN1 identified by ChIP-seq (upper panel). Dyp-30 also binds to the LBAL site, according to Dpy-30 ChIP-seq results deposited in the GEO database under GSE26136 (lower panel). Arrow: LSD1- and Dpy-30-binding peaks in similar location with LBAL site. Scale bar, $5 \mathrm{~kb}$. (b) LBAL sites are conserved in human, mouse and rat. (c) Both LSD1 and dimethyl H3K4 bind to LBAL site, as determined by ChIP of E16.5 rat brain followed by qPCR. ${ }^{\star} P=0.029(\mathrm{LSD} 1, n=3)$; ${ }^{\star} P=0.047$ (dimethyl H3K4, $n=3$; paired $t$-test). (d) LSD1 inhibitor increases H3K4 dimethylation and monomethylation at LBAL. E16.5 rat brains were dissociated and cultured in DMEM/N2/bFGF for $12 \mathrm{~h}$, then treated with saline (Ctrl) or tranylcypromine $(\mathrm{T}, 10 \mu \mathrm{M})$ for $8 \mathrm{~h}$, followed by ChIP with lgG, or corresponding antibodies as indicated. ${ }^{\star \star \star} P<0.001,{ }^{\star \star} P<0.01$, NS, not significant. ( $n=3$, except for dimethyl H3K4, $n=6$; t test). (e,f) LSD1 inhibition suppresses ATN1 expression. NPCs were treated as in $\mathbf{d}$ for $12 \mathrm{~h}$. (e) ATN1 mRNA analysed by qPCR. ${ }^{\star \star \star} P=0.0002(n=14)$. (f) Protein levels determined by western blotting. ${ }^{\star \star} P=0.0001$ ( $n=6$; paired $t$-test). (g) Rat brains were electroporated with shCtrl or shL-1 at E16.5 and DsRED ${ }^{+}$cells were collected by fluorescence-activated cell sorting at E19.5. LSD1 and ATN1 mRNA levels in these cells were analysed by qPCR. ${ }^{\star \star} P=0.002,{ }^{\star \star \star} P<0.001,(n=3 ; t$-test). (h) Protein levels in E14.5 wild-type or LSD1 cKO cortices were determined by western blotting. ${ }^{\star \star \star} P=0.0003(n=3$; $t$-test). All data represent mean \pm s.e.m. 
quantitative PCR (qPCR), which showed enrichment by 3.3- and 23.8-fold, respectively, relative to the IgG control (Fig. 3c). In addition, treatment of cultured cortical progenitors for $8 \mathrm{~h}$ with tranylcypromine increased the levels of both dimethyl and monomethyl H3K4 at LBAL without apparently altering the H3K9 monomethylation at LBAL (Fig. 3d), suggesting that the demethylase activity of LSD1 is normally required to suppress H3K4 methylation at this site. Moreover, both messenger RNA and protein expression of ATN1 was suppressed by tranylcypromine treatment (Fig. 3e,f), corresponding to the observed premature differentiation of NPCs (Fig. 2b). Although the dimethyl (not monomethyl) H3K9 level at LBAL was also enhanced by tranylcypromine treatment, much less histone methylation modification occurred in $\mathrm{H} 3 \mathrm{~K} 9$ than in $\mathrm{H} 3 \mathrm{~K} 4$, suggesting that LBAL site is mostly occupied and regulated by H3K4 methylation modification and not H3K9 methylation (Fig. 3d).

To confirm that LSD1 regulated ATN1 expression in vivo, rat brains were electroporated with shLSD1 at E16.5, and 3 days later those DsRED $^{+}$cells were collected through fluorescenceactivated cell sorting. Consistently, the qPCR results showed the efficient knockdown of LSD1 by shLSD1 and significant downregulation of $A T N 1$ as well (Fig. $3 \mathrm{~g}$ ). This was also confirmed by immunostaining of those cells transfected with shLSD1 in the VZ with both LSD1 and ATN1 antibodies (Supplementary Fig. 6a,b). More importantly, we detected significantly lower ATN1 protein levels in E14.5 cortices of brain-specific LSD1 cKO mice (Fig. 3h). Decreased ATN1 expression was also confirmed in the NPCs residing in the $\mathrm{VZ}$ of $L S D 1 \mathrm{cKO}$ mice cortex by immunostaining (Supplementary Fig. 6c,d). All these in vitro and in vivo results demonstrate that ATN1 expression is positively regulated by LSD1. This finding was unexpected, as $\mathrm{H} 3 \mathrm{~K} 4$ dimethylation is typically associated with transcriptional activation ${ }^{16}$; thus, LSD1 demethylase activity would be predicted to suppress ATN1 expression, while tranylcypromine treatment or LSD1 knockout should have the opposite effect.

Given that LSD1 binds to the LBAL site downstream of ATN1 locus and positively regulates $A T N 1$ expression, we investigated whether the regulation of ATN1 expression by LSD1 is through LBAL. The LBAL sequence $(1.98 \mathrm{~kb})$ was cloned upstream of the enhanced green fluorescent protein (eGFP) reporter in the pCMS-eGFP backbone (pCMS-eGFP-LBAL), becoming a downstream insertion on vector linearization (Supplementary Figs $7 \mathrm{a}$ and 4). Interestingly, the presence of the LBAL downstream of GFP dramatically enhanced the expression of GFP (Supplementary Fig. 7c). Tranylcypromine treatment substantially suppressed GFP expression in cells transfected with pCMS-eGFP-LBAL but not those with pCMS-eGFP (Supplementary Fig. 7b,c), and increased the dimethyl H3K4 level at the LBAL site in the linearized plasmids (Supplementary Fig. 7d). Thus, our results suggest that LSD1 regulates ATN1 expression through the modulation of LBAL methylation status. In addition, the LBAL site may play a role as an enhancer for gene expression and its activity is likely to be regulated by increased H3K4 methylation level.

ATN1 expression is associated with $\mathrm{H} 3 \mathrm{~K} 4$ methylation levels at LBAL. The knockdown of the SET1/MLL histone methyltransferase complex subunits, RbBP5 and Dpy-30, has been reported to direct the differentiation potential of embryonic stem cells along the neural lineage ${ }^{29}$, with concomitant defects in gene repression, including ATN1 (Supplementary Fig. 8). In addition, we have shown above that LSD1 regulates ATN1 expression and NPC differentiation. Thus, we hypothesized that LSD1 could maintain a progenitor state in NPCs by antagonizing methyltransferase activity to regulate ATN1 expression. To test this, the expression of ATN1 in the embryonic brain was examined. ATN1 immunoreactivity was high relative to the nuclear (4',6-diamidino-2-phenylindole) signal in the apical progenitor cells within the VZ, lower in the intermediate/basal progenitors of the SVZ, the lowest in the IZ and higher in neurons in the CP (Fig. 4a,b). Similarly, ATN1 expression in Nestin $^{+}$-cultured NPCs was substantially higher than in Tuj $1^{+}$. differentiated neurons (Fig. 4c,d). These results imply that a downregulation of ATN1 expression is required for differentiation to occur, after which the levels again increase. To test this, basic fibroblast growth factor (bFGF) and epidermal growth factor (EGF) were withdrawn from the culture medium to induce differentiation of NPCs in a neurosphere culture. Within 2-6h of growth factor withdrawal, ATN1 mRNA levels decreased, followed by an increase at $12-36 \mathrm{~h}$, a trend similar to what was observed in vivo (Fig. 4e).

To test whether the histone methylation levels at LBAL site was associated with the regulation of ATN1 expression during NPC differentiation, neurosphere cultures were subjected to growth factor withdrawal, to induce differentiation, and different histone methylation modification levels at LBAL were evaluated by ChIPqPCR. As shown in Fig. 4f, H3K4 dimethylation levels at LBAL increased very significantly at $6 \mathrm{~h}$ after growth factor withdrawal but dropped relatively at $36 \mathrm{~h}$, corresponding to the expression of ATN1 (Fig. 4e). Meanwhile, the H3K4 and H3K9 monomethylation levels were not apparently changed. Although the dimethyl H3K9 levels increased $6 \mathrm{~h}$ after growth factor withdrawal, similar to tranylcypromine-treated NPCs (Fig. 3d), it's enrichment was also much lower than that of $\mathrm{H} 3 \mathrm{~K} 4$.

To investigate the role of LBAL in the regulation of ATN1 expression in vivo, linerated pCMS-eGFP or pCMS-eGFP-LBAL together with control or shLSD1 were introduced into NPCs of E16.5 rat brain via IUE and were analysed at E19.5. As shown in Supplementary Fig. 9, the GFP levels in the cells expressing pCMS-eGFP were similar in the VZ and SVZ (Supplementary Fig. 9a). It was lower in the IZ, probably due to the dilution of plasmid during cortical development. However, the GFP levels in the cells expressing pCMS-eGFP-LBAL were downregulated significantly in both SVZ and IZ, reminiscent of the endogenous ATN1 levels (Fig. 4a and Supplementary Fig. 9b), suggesting the role of $\mathrm{LBAL}$ in the regulating ATN1 expression in vivo. Interestingly, when LSD1 was knocked down, the difference of GFP levels in the VZ and SVZ was compromised (Supplementary Fig. 9c), similar to the compromised expression of endogenous ATN1 in the VZ and SVZ of LSD1 cKO mouse cortex (Supplementary Fig. 6c,d). These indicate that LSD1 regulates ATN1 expression through the control of the histone methylation status at LBAL.

ATN1 knockdown in NPCs leads to premature differentiation. Given that LSD1 regulates ATN1 expression, which is downregulated during NPC differentiation, the effects of ATN1 depletion on progenitor maintenance was examined. Two shRNA/eGFP constructs which were highly efficient at suppressing exogenous and endogenous ATN1 expression (Fig. 5c and Supplementary Fig. 10a), were electroporated into E16.5 embryos and examined 4 days later. Their effects were similar to those observed on LSD1 knockdown; that is, fewer transfected cells were observed in the proliferative zones compared with controls, and cells failed to reach the CP (Fig. 5a,b). Co-transfection of a shRNA-resistant human ATN1 construct rescued the phenotype (Supplementary Fig. 11), although a slight delay in migration was still observed. In addition, no apparent increase of cell death was detected in ATN1 knockdown cells (Supplementary Fig. 10b). 
a
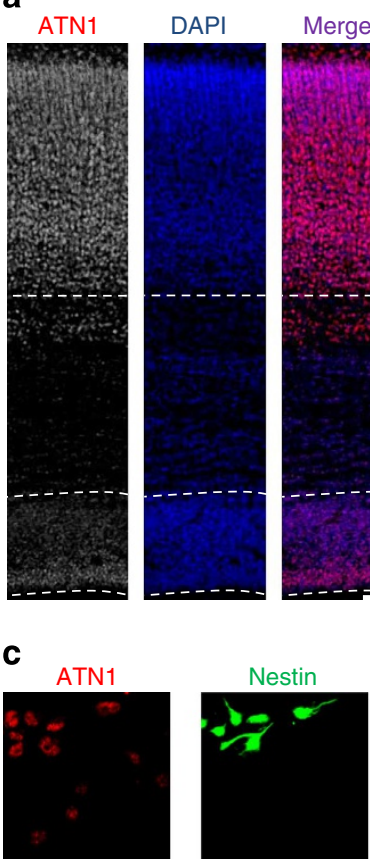

c
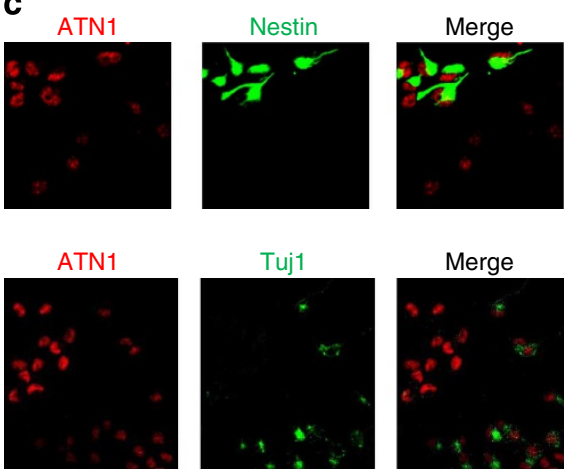

Merge

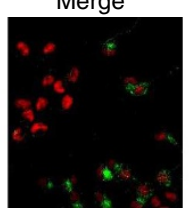

ATN1

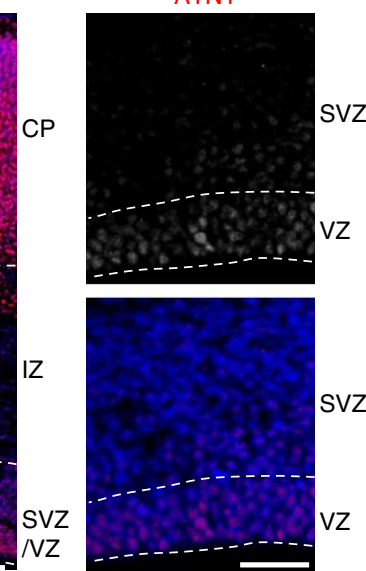

b

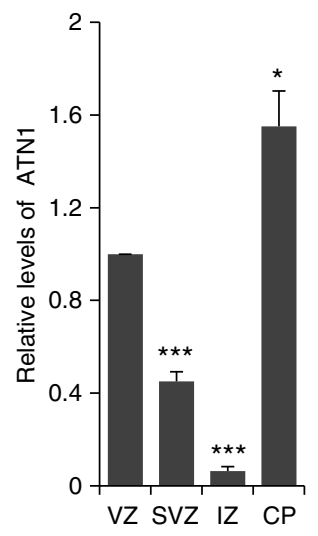

d

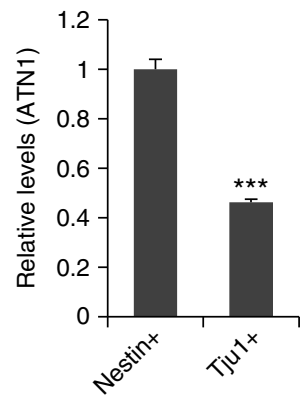

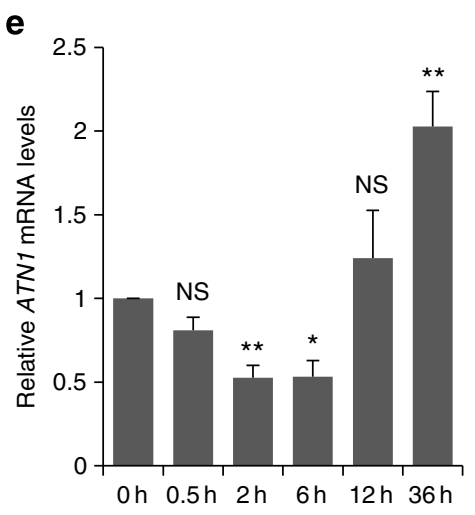

f

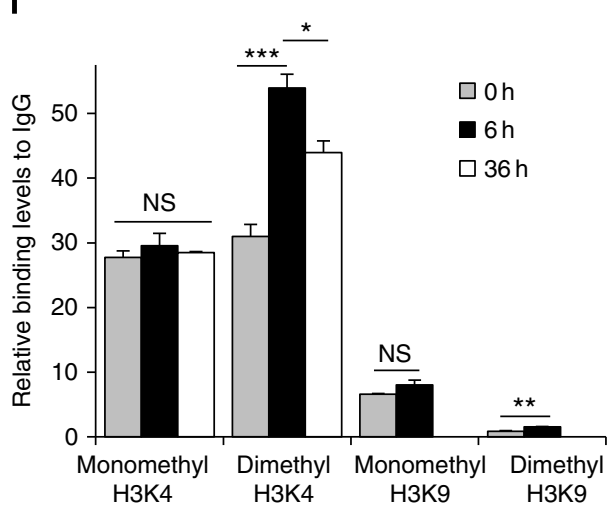

Figure 4 | ATN1 expression is downregulated during NPC differentiation. (a) E20.5 rat coronal cortical sections stained with ATN1 antibody and 4',6-diamidino-2-phenylindole (DAPI). Right panels show high-magnification view of the VZ and SVZ. Scale bar, $50 \mu \mathrm{m}$. (b) Quantification of ATN1 levels (normalized to DAPI) in the VZ, SVZ, IZ and CP. ${ }^{\star \star \star} P<0.001,{ }^{\star} P=0.015$, ( $n=6 / 3$; paired $t$-test). $n$, number of sections from different brains. (c) E16.5 rat brains were dissociated and cultured in DMEM/B27/bFGF/EGF/P/S for 48 h, fixed, then immunostained for ATN1 and Nestin (upper panel) or Tuj1 (lower panel) and DAPI. Scale bar, $20 \mu \mathrm{m}$. (d) Quantification of ATN1 levels in Nestin ${ }^{+}$and Tuj1 ${ }^{+}$cells. ${ }^{\star \star \star} P<0.001$ (Nestin, $n=50$; Tuj1, $n=50$; Student's t-test). (e) Quantification of ATN1 mRNA levels in neurospheres at time indicated after bFGF and EGF withdrawal by real-time PCR. ${ }^{\star} P=0.017,{ }^{\star \star} P=0.0076(2 \mathrm{~h}),{ }^{\star \star} P=0.0081$ (36h). (0.5 h, $n=4 ; 2 \mathrm{~h}, n=4 ; 6 \mathrm{~h}, n=4 ; 12 \mathrm{~h}, n=7 ; 36 \mathrm{~h}, n=5$; paired $t$-test). NS, not significant. (f) Neurospheres from dissociated E16.5 rat brains were cultured in DMEM/B27/bFGF/EGF for $36 \mathrm{~h}$ before bFGF and EGF withdrawal as in e; 6 or $36 \mathrm{~h}$ later, different histone modification levels at LBAL were analysed as in Fig. 3d. ${ }^{\star} P=0.024,{ }^{\star \star} P=0.0028,{ }^{\star \star \star} P<0.001$ ( $n=3$; except for dimethyl H3K4 in $6 \mathrm{~h}, n=6$; one-way analysis of variance). All data are means \pm s.e.m.

The transfected cells were examined for cell fate marker expression to determine whether the loss of cells in the VZ and SVZ was due to premature differentiation of NPCs. The fraction of ATN1-deficient cells expressing the progenitor markers Sox2 and Tbr2 was dramatically lower than for control-transfected cells, while the fraction of cells expressing the neuronal protein Tuj1 was significantly higher (Fig. $5 \mathrm{~d}$ ). These results indicate that
ATN1 knockdown leads to the depletion of the NPC population as a result of premature differentiation.

LSD1 promotes NPC maintenance via regulation of ATN1. Given that depletion of ATN1 and LSD1 both induce premature differentiation, we went on to investigate whether the 
a
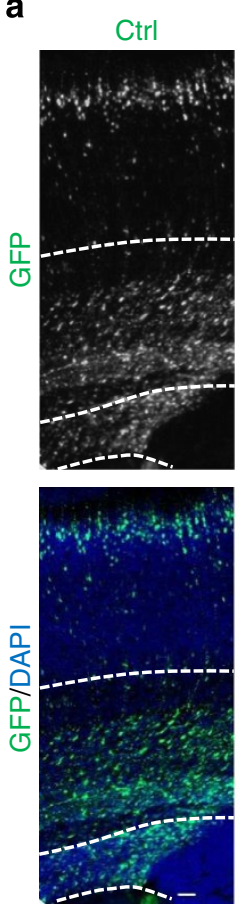

d
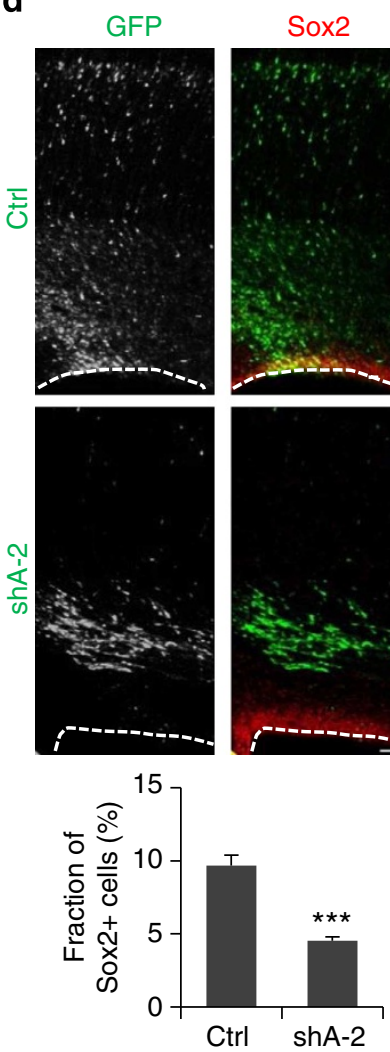

$\operatorname{shA}-1$
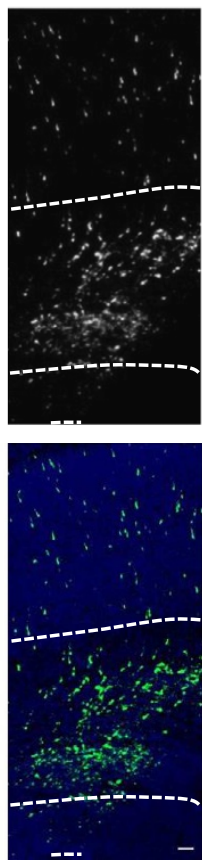

Sox2
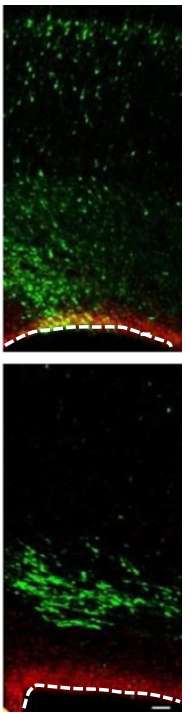

Ctrl shA-2
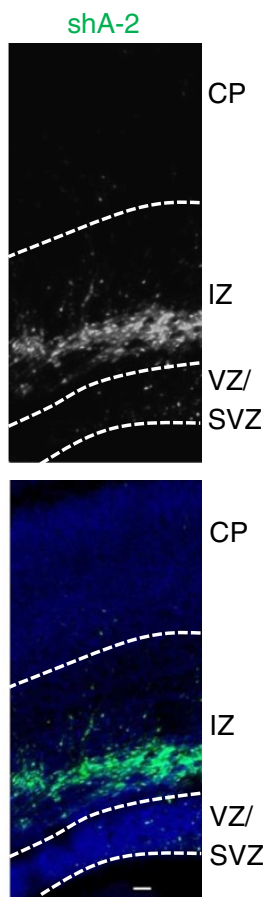

GFP
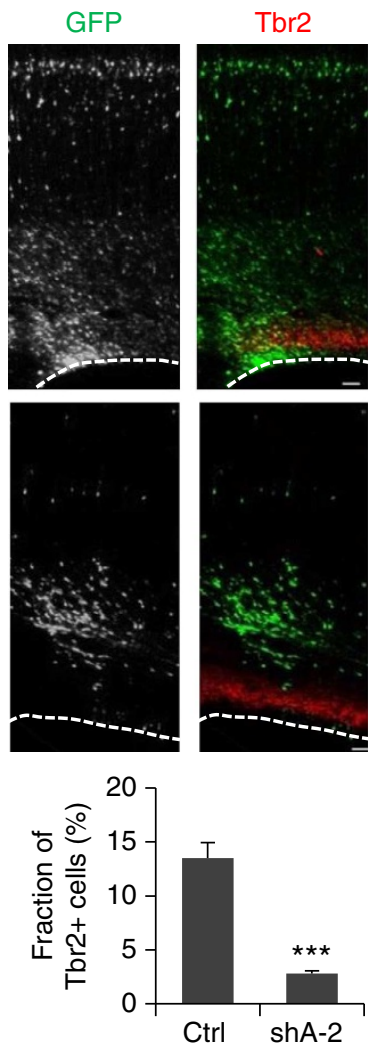

C b
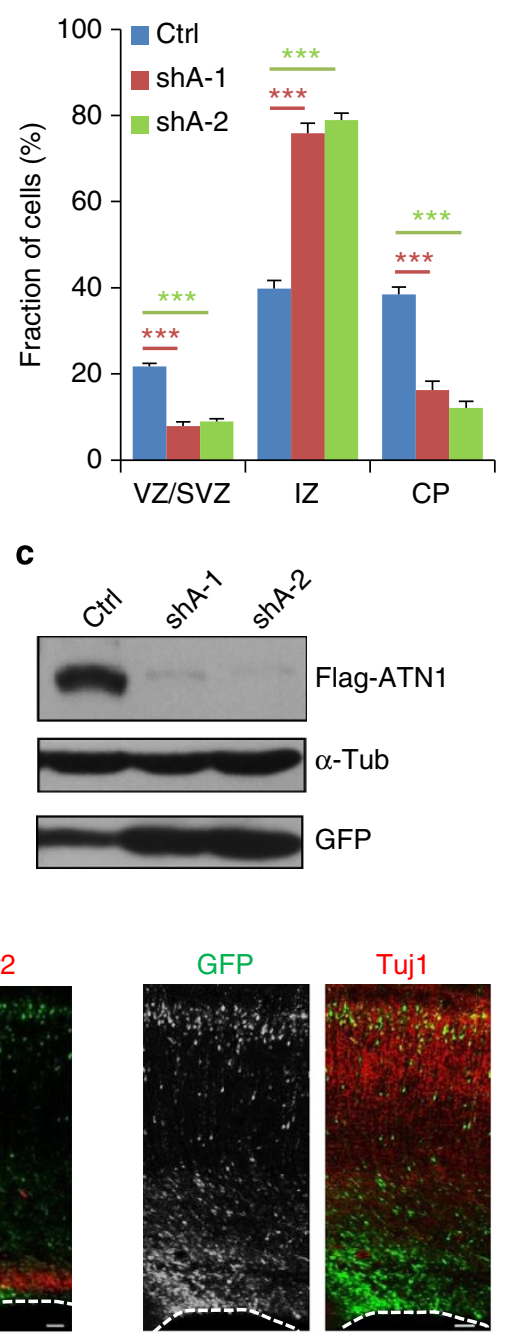

Tuj1
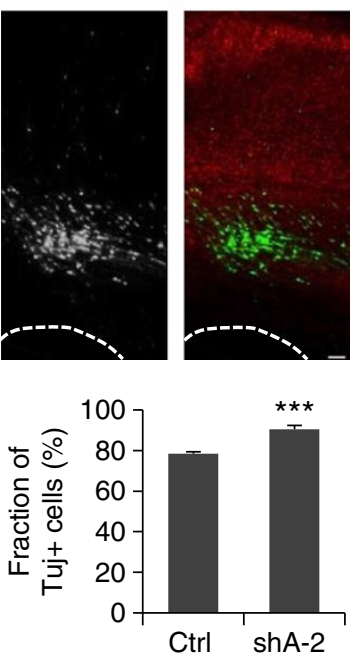

Figure 5 | ATN1 knockdown phenocopies the depletion of LSD1. (a,b) ATN1 knockdown was performed as for LSD1 (Fig. 1d). Data represent mean \pm s.e.m. ${ }^{\star \star \star} P<0.001$ (Ctrl, $n=21 / 7$; shA-1, $n=12 / 4$; shA-2, $n=27 / 9$; one-way analysis of variance). (c) ATN1 can be efficiently knocked down by ATN1 shRNAs (shA). Flag-ATN1 was co-transfected with shCtrl or shAs into HEK293 cells; $24 \mathrm{~h}$ later, cell lysates were analysed by immunoblotting with anti-ATN1 antibody, with $\alpha$-tubulin serving as loading control and GFP as transfection efficiency control. (d) ATN1 knockdown leads to premature differentiation of NPC. Data represent mean \pm s.e.m. ${ }^{\star \star \star} P<0.001$. Sox2 (Ctrl, $n=5 / 5 ;$ shA-2, $\left.n=5 / 5\right)$; Tbr2 (Ctrl, $\left.n=4 / 4 ; \operatorname{shA}-2, n=6 / 6\right) ;$ Tuj1 (Ctrl, $n=5 / 5 ;$ shA-2, $n=7 / 7$ ); Student's t-test. $n$, number of sections from different brains. Scale bar, $50 \mu \mathrm{m}$. 
maintenance of NPCs controlled by LSD1 depends on the regulation of ATN1. E16.5 embryos were simultaneously electroporated with LSD1 shRNA and full-length ATN1 or control vector. The overexpression of ATN1 rescued the depletion of NPCs in the VZ and SVZ caused by LSD1 knockdown (Fig. 6a,b). More importantly, expression of ATN1 rescued the LSD1 knockdown-induced reduction of Sox $2^{+}$and Tbr2 ${ }^{+}$cells, and increase of Tuj ${ }^{+}$cells (Fig. 6c), indicating that the loss of LSD1 in NPCs induces their premature differentiation into neurons through the deregulation of ATN1 expression. Thus, we would like to propose that the demethylation of LBAL site downstream of ATN1 locus by LSD1 maintains the pool of cortical progenitors through the induction of ATN1 expression. Inhibition, knockdown or knockout of LSD1, as well as ATN1 depletion, causes NPCs to differentiate prematurely (Fig. 7).

\section{Discussion}

Histone modification is a critical mechanism of gene regulation during development. Here we provide evidence that the demethylase LSD1 and its target gene ATN1 are responsible for NPC maintenance during cortical development in vivo, providing another example of how specific epigenetic marks can direct cell fate choices. Premature NPC differentiation was associated with increased $\mathrm{H} 3 \mathrm{~K} 4$ dimethylation at LBAL, the LSD1-binding site of $A T N 1$ and lower levels of ATN1 expression (Figs 2 and 3). Conversely, NPCs expressed ATN1 at high levels, but had relatively low levels of dimethyl H3K4 (Fig. 4). These changes in methylation status are probably achieved through the antagonistic activities of a methyltransferase and LSD1. A reduction in demethylase activity by LSD1 knockdown, knockout or by treatment with a LSD1 inhibitor ${ }^{18}$, would lead to increased methylation at LBAL and downregulation of ATN1 expression, resulting in NPC differentiation (model in Fig. 7).

A candidate methyltransferase in this model is the SET1/MLL histone methyltransferase complex. This is supported by two lines of evidence (Fig. 3a and Supplementary Fig. 8). First, depletion of RbBP5 and Dpy30, two subunits of the complex, produces an effect opposite to LSD1 knockdown; namely, a defect in ATN1 repression during differentiation and thus the restriction of stem cell differentiation potential along the neural lineage ${ }^{29}$. Second, both Dpy30 and LSD1 bind to the LBAL locus ${ }^{29}$. Future studies can examine whether inhibiting the activity of the SET1/MLL complex can alter methylation at LBAL in cortical progenitors and induce an expansion of the progenitor pool.

Although we cannot exclude the possibility that LSD1 regulates ATN1 expression levels through an indirect effect of blocking global histone demethylation, it is very likely to be that LSD1 regulates ATN1 expression via controlling the histone methylation levels at LBAL. First, the expression of reporter gene with a downstream LBAL site can be suppressed by tranylcypromine treatment, similar to that of endogenous ATN1 (Fig. 3 and Supplementary Fig. 7). Second, reporter gene expression in cells transfected with pCMS-eGFP-LBAL is significantly lower in the SVZ than that in the VZ and this is compromised by LSD1 knockdown (Supplementary Fig. 9). Thus, it is reasonable to deduce that the downregulation of LSD1 level during NPC differentiation in the SVZ (Fig. 1a) ${ }^{18}$ leads to increased histone methylation levels at LBAL, and thus the corresponding decreased ATN1 expression in the SVZ as that of LSD1 (Figs 3,4).

LSD1 has been shown to remove both the mono- or dimethylation of $\mathrm{H} 3 \mathrm{~K} 4$ and $\mathrm{H} 3 \mathrm{~K} 9$ (refs 13,30). Although we are not certain that downregulation of ATN1 expression by LSD1 inhibition is caused by increased monomethyl H3K4 or dimethyl $\mathrm{H} 3 \mathrm{~K} 9$ at the LBAL site, this kind of regulation is more likely to be mediated by dimethyl $\mathrm{H} 3 \mathrm{~K} 4$ for two reasons: One is that the H3K9 methylation levels at LBAL site in NPCs are much lower compared with that of H3K4 methylation (Figs 3d,4f); The other reason is that when NPCs undergo differentiation, it is changes of dimethyl H3K4, but not monomethyl H3K4 levels, that are accompanied by the alternation of ATN1 expression (Fig. 4f).

Although H3K4 trimethylation in promoter region has been observed to repress transcription ${ }^{31}$, tri- and dimethylated H3K4 are predominantly associated with the activation of gene expression ${ }^{6}$. This maybe the reason why LSD1 is mostly reported as a transcriptional repressor ${ }^{16,32}$. In the present study, inhibition of LSD1 activity resulted in enhanced H3K4 dimethylation at LBAL in NPCs, accompanied by the downregulation of ATN1 expression. It is interesting to speculate that this contradictory effect is due to the location of LBAL downstream of the ATN1 coding region, instead of within the promoter as is typically the case. In this instance, a high dimethyl H3K4 level at LBAL might produce a conformational change in the chromatin that would favour transcriptional repression, perhaps by physically obstructing the access of the transcriptional complex to the promoter. Although LSD1 activates androgen receptor transcription by removal of monoand dimethyl H3K9 (instead of H3K4) histone marks ${ }^{14,30}$, to the best of our knowledge our study is likely to be the first report that LSD1-involved H3K4 methylation is required for gene activation as well as NPC maintenance.

Even though the present results indicate that $A T N 1$ is probably a direct target of LSD1 in the regulation of NPC maintenance, there are some aspects of the mechanism that remain unclear. For instance, ATN1 has been reported to interact with and modulate the repressor activity of TLX ${ }^{32}$, while loss of TLX function also induces premature differentiation of $\mathrm{NPCs}^{33}$, raising the possibility that ATN1 exerts its effect on progenitor fate through TLX. LSD1 and TLX have also been shown to directly interact and coordinately repress target gene expression ${ }^{16,32}$. Therefore, LSD1 could regulate ATN1 directly, or else indirectly through TLX. Alternatively, LSD1, ATN1 and TLX could interact within a single complex. Reduced levels of ATN1 could disrupt the repressor complex that normally suppresses differentiation and maintains progenitors in a state of self-renewal, resulting in the derepression of differentiation-promoting genes.

In addition to the epigenetic regulation of $A T N 1$ by LSD1 during embryonic brain development, ATN1 expression in the adult brain would be investigated in the future to determine whether it can be suppressed by the LSD1 inhibitor tranylcypromine, which is currently prescribed for the treatment of mood and anxiety disorders in the clinic. If that is the case, given that DRPLA is caused by the aggregation of ATN1, tranylcypromine could be tried for the prevention and alleviation of some DRPLA symptoms.

\section{Methods}

Plasmids. LSD1-specific shRNAs, shL-1 and shL-2 were constructed into RetroQ-DsRed-shRNA. Target sequences (for rat LSD1) of shControl: $5^{\prime}$-GTG CGTTGCTAGTACCAAC- $3^{\prime}$; shL-1: $5^{\prime}$-GGCGAAGGTAGAATACAGA-3'; shL-2: $5^{\prime}$-GGATGGATGTCACACTTCT-3'. ATN1-specific shRNAs, shA-1 and shA-2, were cloned into pLL3.7-GFP. Target sequences (for rat ATN1) of shcontrol: 5'-TCATGCCTCTATCTACGTC-3'; shA-1: 5'-CGAGAACGAAGTTCTTCGT-3'; shA-2: $5^{\prime}$-GGGCCTTCAAGTTTGTCAT-3'. Flag-tagged human ATN1 were from Dr Chih-Cheng Tsai ${ }^{34}$, and were recloned into pCAGIG. Rat ATN1 was amplified by PCR from E16.5 rat brain complementary DNA and cloned into pCMV-Tag2b. Flag-tagged human LSD1 was from Dr Zengqiang Yuan. Human LSD1 was synonymously mutated into a form refractory to shL-1 and cloned into pCAGIG (from $5^{\prime}$-ggcgaaggtagagtacaga- $3^{\prime}$ to $5^{\prime}$-ggcCaaAgtGgagtacaga- $3^{\prime}$ ). Rat LSD1 ATN1 was amplified by PCR from E16.5 rat brain cDNA and cloned into peGFP-N1. The LSD1-binding site around ATN1 locus was cloned from rat genome and inserted into the EcoR1/Sal1 site of pCMS-eGFP. The primers used for cloning are listed in Supplementary Table 2. 
a
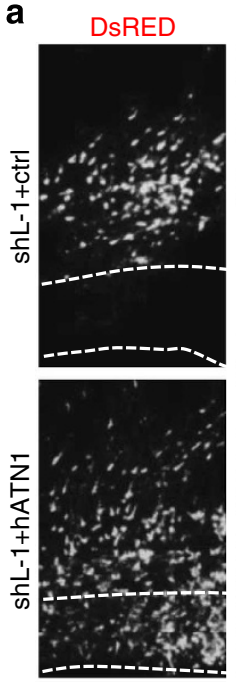

C
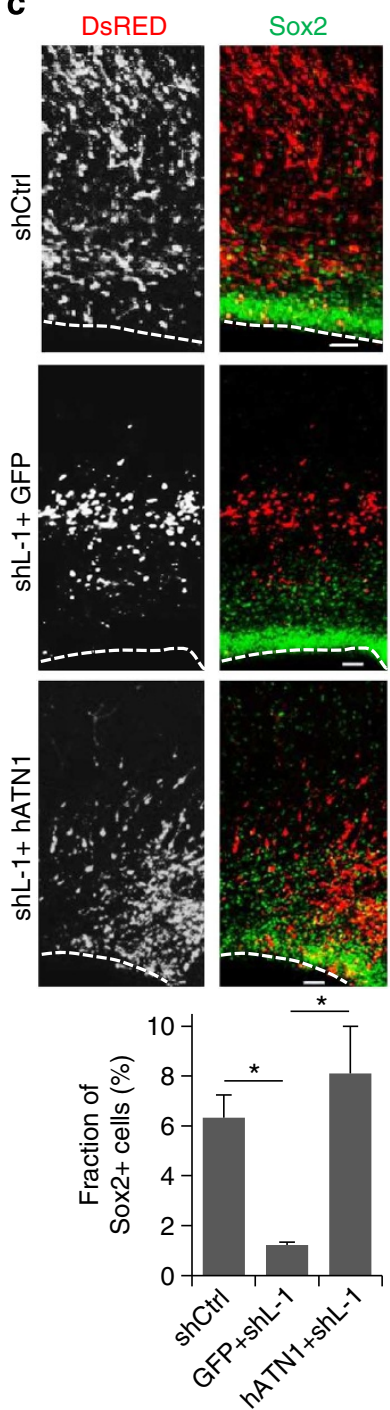

GFP
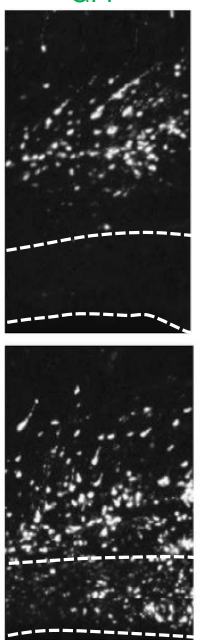

*i:

b
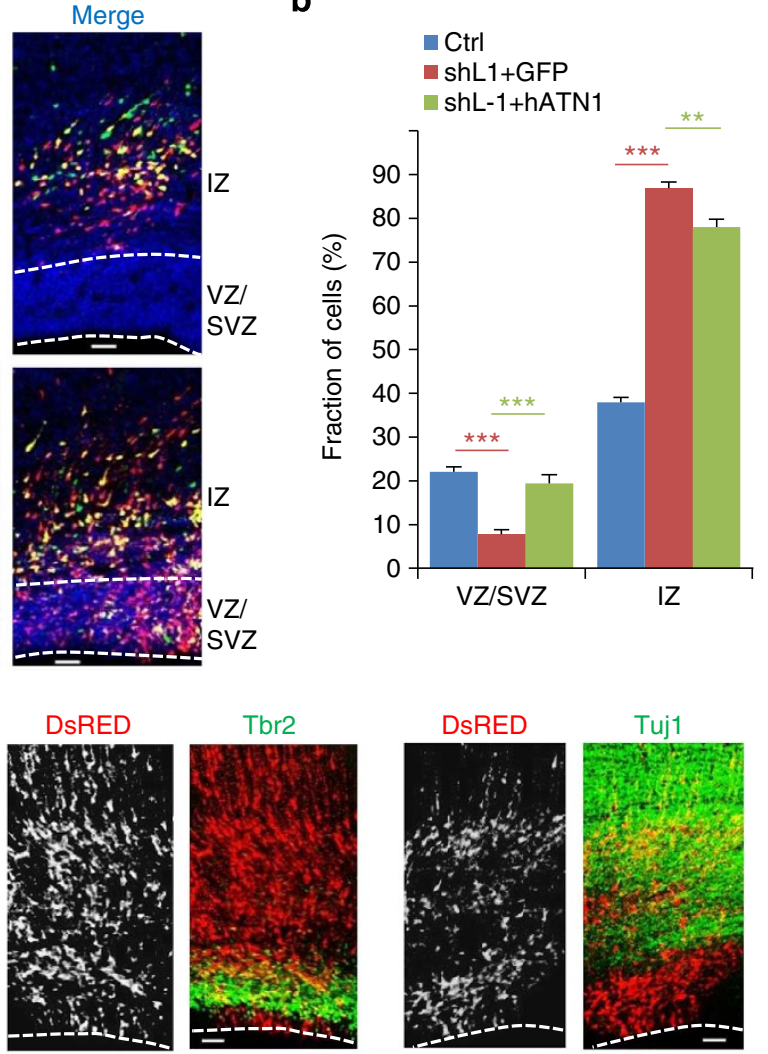

Tuj1
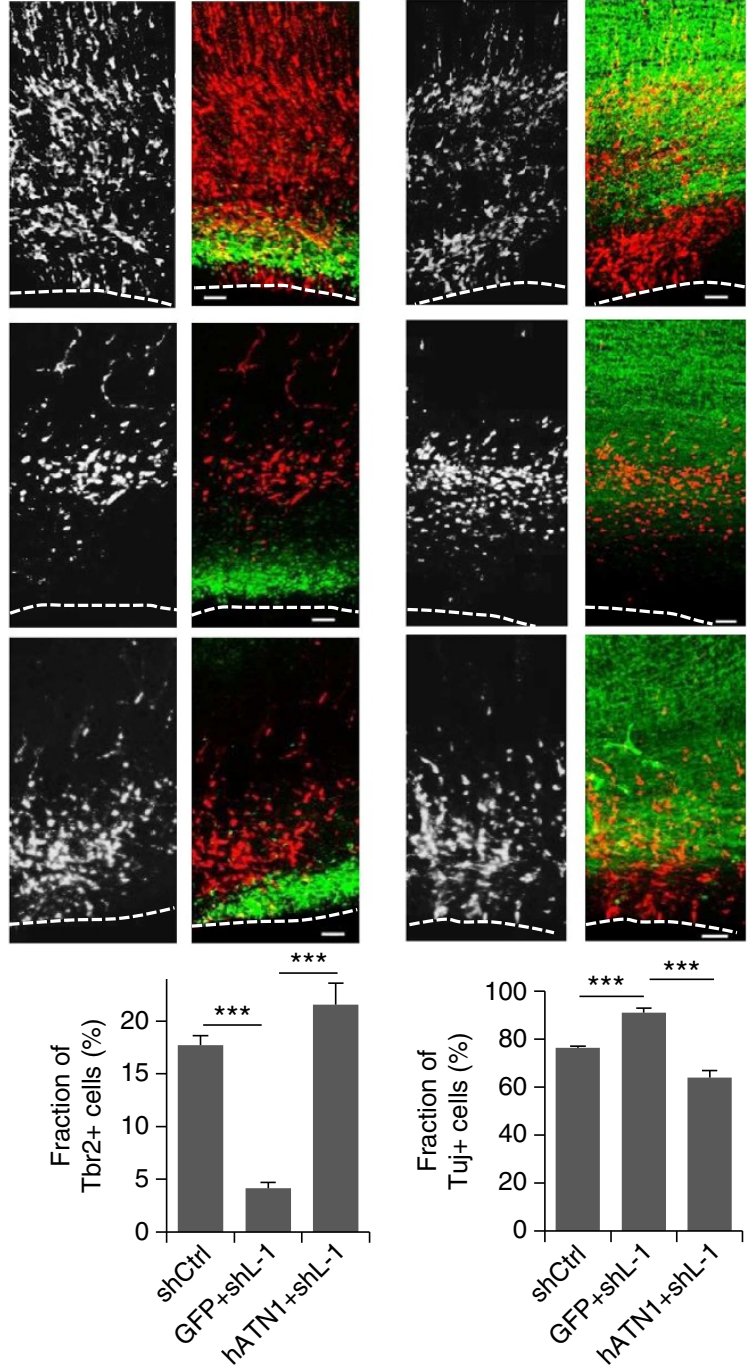

Figure 6 | LSD1 maintains NPCs in a progenitor state via regulation of ATN1. (a) ATN1 overexpression rescues shLSD1-induced NPC depletion in the VZ and SVZ. Coronal sections of rat brains electroporated with shL-1 along with pCAGIG or pCAGIG-hATN1 at E16.5 and examined at E20.5. Scale bar, $50 \mu \mathrm{m}$. (b) Quantification of cell distribution in a. Data are means \pm s.e.m. ${ }^{\star \star} P<0.01$, ${ }^{\star \star \star} P<0.001$ (Ctrl, $n=26 / 8$; shL-1+pCAGIG, $n=8 / 3$; shL-1+pCAGIGhATN1, $n=10 / 3$; one-way analysis of variance (ANOVA)). (c) Rescue experiments were done as in a and analysed as in (Fig. 5d). Data are means \pm s.e.m. ${ }^{\star} P<0.05 ;{ }^{\star \star \star} P<0.001$. Ctrl (Sox2: $n=7 / 5 ;$ Tbr2: $n=5 / 5 ;$ Tuj1: $n=7 / 5$ ); shL-1+pCAGIG (Sox2: $n=3 / 3 ;$ Tbr2: $n=3 / 3 ;$ Tuj1: $n=3 / 3$ ); shL-1+ pCAGIGhATN1 (Sox2: $n=3 / 3$; Tbr2: $n=3 / 3$; Tuj1: $n=3 / 3$ ). one-way ANOVA. $n$, number of brain slices from different brains $(\mathbf{b}, \mathbf{c})$. Scale bar, $50 \mu m$. 


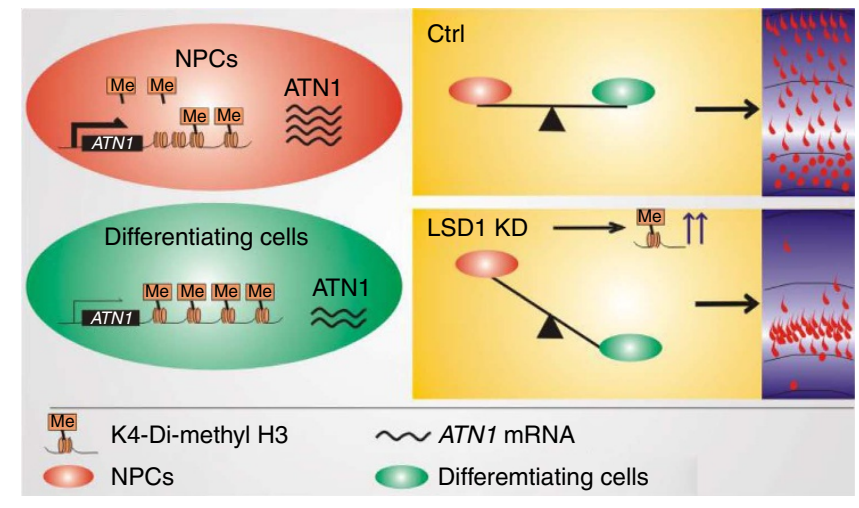

Figure 7 | Model of NPC maintenance by epigenetic regulation of ATN1 by LSD1. In cycling NPCs, LSD1 maintains a low level of H3K4 dimethylation at the LBAL site of ATN1, resulting in a high level of ATN1 expression. In differentiating NPCs, H3K4 dimethylation at LBAL is increased, probably due to antagonistic methyltransferase activity, leading to decreased ATN1 expression and neuronal differentiation. When LSD1 demethylase activity is lost due to knockdown, knockout or tranylcypromine treatment, NPCs prematurely differentiate into neurons.

Cell culture and transfection. HEK293 cells and N2a cells were cultured in DMEM (neuronbc) with $10 \%$ fetal bovine serum (Excel) and 1\% penicillin/streptomycin (P/S, Gibco), and plasmids were transfected into 293 cells with VigoFect (Vigorous) and into N2a cells with Lipo2000 (Invitrogen).

E16.5 rat primary NPCs were cultured in dishes pre-coated with poly-D-lysine and in the DMEM medium with 1\% B27 supplement (Gibco), bFGF (20 $\mathrm{ng} \mathrm{ml}^{-1}$, $\mathrm{R} \& \mathrm{D}), \mathrm{EGF}\left(20 \mathrm{ng} \mathrm{ml}^{-1}, \mathrm{R} \& \mathrm{D}\right), 1 \% \mathrm{P} / \mathrm{S}$ or DMEM with $1 \% \mathrm{~N} 2$ supplement (Gibco), bFGF and 1\% P/S. Neurospheres were cultured as described ${ }^{35}$. Briefly, E16.5 rat brains were dissected and digested with $0.25 \%(\mathrm{~W} / \mathrm{V})$ Trypsin-EDTA for 20 min and then disassociated and cultured in DMEM with 1\% B27 supplement, bFGF, EGF and $1 \% \mathrm{P} / \mathrm{S}$.

Western blotting and immunostaining. Western blotting and immunostaining were performed as described ${ }^{36,37}$. Briefly, the protein samples were separated by SDS-PAGE and transferred to nitrocellulose membranes. The nitrocellulose membrane was incubated with the primary antibody at $4{ }^{\circ} \mathrm{C}$ overnight and then with the horseradish peroxidase-conjugated secondary antibody for $1 \mathrm{~h}$. Pierce ECL (enhanced chemiluminescence) western blotting substrate was used to visualizing the protein bands. The antibodies used for western blotting were as follows: GFP (Abcam, ab290, 1:2,000), $\alpha$-tubulin (CST, 3873s, 1:2,000), GAPDH (CST, 2118s, 1:2,000), LSD1 (CST, 2139s, 1:1,000), ATN1 (Sigma, HPA031619, 1:1,000), Flag (MBL, M185, 1:2,000) and DsRed (MBL, PM005, 1:1,000). For immunostaining experiment, cultured cells or brain slice were fixed with $4 \%$ paraformaldehyde. After incubation with the primary antibody at $4{ }^{\circ} \mathrm{C}$ overnight and then with fluorescent-conjugated secondary antibody for $1 \mathrm{~h}$, images were acquired with Zeiss LSM700. The antibodies used for immunostaining were as follows: LSD1 (CST, 2184s, 1:400), ATN1 (Sigma, HPA031619, 1:400), Sox2 (Abcam, ab97959, 1:1,000), Tbr2 (Millipore, ab2283, 1:1,000), $\beta$-III Tubulin (Abcam, ab7751, 1:1,000), Nestin (Abcam, ab6142, 1:1,000), GFP (Abcam, ab13970, 1:1,000), DsRed (MBL, PM005, 1:1,000) and activated-caspase3 (Abcam, ab13847, 1:1,000). Images of uncropped western blottings are shown in Supplementary Fig. 12.

Animals and in utero electroporation. $L S D 1^{f l o x / f l o x}$ mice were kindly provided by Dr M Rosenfeld's group and Nestin-Cre mice were purchased from Model Animal Research Center of Nanjing University. Pregnant Sprague-Dawley rats and adult ICR female mice were provided by the animal centre of the Institute of Genetics and Developmental Biology (IGDB), Chinese Academy of Sciences, and the experimental procedures were performed according to protocols approved by the Institutional Animal Care and Use Committee at IGDB.

IUE was performed as we described previously ${ }^{38}$. Briefly, the plasmids were injected into the lateral ventricle of E15.5 or E16.5 rat brains, which were given an electrical stimulation ( $50 \mathrm{~V}$ for $50 \mathrm{~ms}$ with a $950 \mathrm{~ms}$ interval) for 5 times. Three or 4 days later, the rats were killed and embryonic brains were fixed in $4 \%$ paraformaldehyde and dehydrated in $30 \%$ sucrose. The brains were frozensectioned into 50- $\mu \mathrm{m}$ slices with Leica CM1950.

Imaging and statistical analysis. Confocal images were achieved through Zeiss LSM700 and analysed with Photoshop, ImageJ or Imaris. The data were analysed with $t$-test, paired $t$-test or one-way analysis of variance and the significant difference was shown as ${ }^{*} P<0.05,{ }^{*} P<0.01$ and ${ }^{* * *} P<0.001$. The fraction of Sox $2+$ cells (and Tbr $2+$ and Tuj $1+$ cells) was calculated by dividing number of all cells transfected with shRNAs by number of Sox $2+$ (and Tbr $2+$ and Tuj $1+$ cells) cells transfected with shRNAs across the cortex. The number of brain slices analysed was displayed in ' $n=a / b$ ', where ' $a$ ' is the total number of brain slices analysed and ' $b$ ' is the number of different mouse or rat brains.

RNA extraction and real-time PCR. RNA extraction with TRIZOL was performed as described ${ }^{39}$. Cultured cells or brain tissues were dissolved in $1 \mathrm{ml}$ TRIZOL and $200 \mu \mathrm{l}$ chloroform was then added. After precipitation with isopropanol and washing with $70 \%(\mathrm{~V} / \mathrm{V})$ ethanol, the collected mRNA was reversely transcribed into cDNA with reverse transcriptase (Promega), and realtime PCR was performed with Bio-Rad C1000 Thermal Cycler. The primers used are listed in Supplementary Table 2.

ChIP and ChIP-seq. ChIP with LSD1 antibody (CST, 2184s) and Di-MethylHistone H3 (K4) antibody (CST, 9726s) was performed with SimpleChIP Kit (CST) according to manufacturer's protocol. Purified DNA from ChIP samples with LSD1 and IgG antibodies from E16 rat brains were then sent to Beijing Genomics Institute (BGI, China) for sequencing and data analyses. Our ChIP-Seq data has been deposited in the GEO database under the accession number of GSE62770.

\section{References}

1. Lopez-Otin, C., Blasco, M. A., Partridge, L., Serrano, M. \& Kroemer, G. The hallmarks of aging. Cell 153, 1194-1217 (2013).

2. Iacobuzio-Donahue, C. A. Epigenetic changes in cancer. Annu. Rev. Pathol. 4, 229-249 (2009).

3. Papp, B. \& Plath, K. Epigenetics of reprogramming to induced pluripotency. Cell 152, 1324-1343 (2013).

4. Tan, L. \& Shi, Y. G. Tet family proteins and 5-hydroxymethylcytosine in development and disease. Development 139, 1895-1902 (2012).

5. Hirabayashi, Y. \& Gotoh, Y. Epigenetic control of neural precursor cell fate during development. Nat. Rev. Neurosci. 11, 377-388 (2010).

6. Li, B., Carey, M. \& Workman, J. L. The role of chromatin during transcription. Cell 128, 707-719 (2007).

7. Lefevre, G. M., Patel, S. R., Kim, D., Tessarollo, L. \& Dressler, G. R. Altering a histone H3K4 methylation pathway in glomerular podocytes promotes a chronic disease phenotype. PLoS Genet. 6, e1001142 (2010).

8. Greer, E. L. \& Shi, Y. Histone methylation: a dynamic mark in health, disease and inheritance. Nat. Rev. Genet. 13, 343-357 (2012).

9. Buchman, J. J., Durak, O. \& Tsai, L. H. ASPM regulates Wnt signaling pathway activity in the developing brain. Genes Dev. 25, 1909-1914 (2011).

10. Ayala, R., Shu, T. \& Tsai, L. H. Trekking across the brain: the journey of neuronal migration. Cell 128, 29-43 (2007).

11. Huttner, W. B. \& Kosodo, Y. Symmetric versus asymmetric cell division during neurogenesis in the developing vertebrate central nervous system. Curr. Opin. Cell Biol. 17, 648-657 (2005).

12. Chenn, A. \& Walsh, C. A. Regulation of cerebral cortical size by control of cell cycle exit in neural precursors. Science 297, 365-369 (2002).

13. Shi, Y. et al. Histone demethylation mediated by the nuclear amine oxidase homolog LSD1. Cell 119, 941-953 (2004).

14. Wang, J. et al. Opposing LSD1 complexes function in developmental gene activation and repression programmes. Nature 446, 882-887 (2007).

15. Fuentes, P., Canovas, J., Berndt, F. A., Noctor, S. C. \& Kukuljan, M. CoREST/ LSD1 control the development of pyramidal cortical neurons. Cereb. Cortex 22, 1431-1441 (2012).

16. Sun, G. et al. Histone demethylase LSD1 regulates neural stem cell proliferation. Mol. Cell Biol. 30, 1997-2005 (2010).

17. Adamo, A. et al. LSD1 regulates the balance between self-renewal and differentiation in human embryonic stem cells. Nat. Cell Biol. 13, 652-659 (2011).

18. Sun, G. et al. miR-137 forms a regulatory loop with nuclear receptor TLX and LSD1 in neural stem cells. Nat. Commun. 2, 529 (2011).

19. Naito, H. \& Oyanagi, S. Familial myoclonus epilepsy and choreoathetosis: hereditary dentatorubral-pallidoluysian atrophy. Neurology 32, 798-807 (1982).

20. Oyanagi, S. Hereditary dentatorubral-pallidoluysian atrophy. Neuropathology 20(Suppl): S42-S46 (2000).

21. Suzuki, Y. \& Yazawa, I. Pathological accumulation of atrophin-1 in dentatorubralpallidoluysian atrophy. Int. J. Clin. Exp. Pathol. 4, 378-384 (2011).

22. Zhang, C. L., Zou, Y., Yu, R. T., Gage, F. H. \& Evans, R. M. Nuclear receptor TLX prevents retinal dystrophy and recruits the corepressor atrophin1. Genes Dev. 20, 1308-1320 (2006).

23. Shen, Y., Lee, G., Choe, Y., Zoltewicz, J. S. \& Peterson, A. S. Functional architecture of atrophins. J. Biol. Chem. 282, 5037-5044 (2007).

24. Miller, F. D. \& Gauthier, A. S. Timing is everything: making neurons versus glia in the developing cortex. Neuron 54, 357-369 (2007) 
25. Okano, H. \& Temple, S. Cell types to order: temporal specification of CNS stem cells. Curr. Opin. Neurobiol. 19, 112-119 (2009).

26. Xu, D., Zhang, F., Wang, Y., Sun, Y. \& Xu, Z. Microcephaly-associated protein WDR62 regulates neurogenesis through JNK1 in the developing neocortex. Cell Rep. 6, 104-116 (2014).

27. Lee, M. G., Wynder, C., Schmidt, D. M., McCafferty, D. G. \& Shiekhattar, R. Histone $\mathrm{H} 3$ lysine 4 demethylation is a target of nonselective antidepressive medications. Chem. Biol. 13, 563-567 (2006).

28. Zhang, J. et al. SFMBT1 functions with LSD1 to regulate expression of canonical histone genes and chromatin-related factors. Genes Dev. 27, 749-766 (2013).

29. Jiang, H. et al. Role for Dpy-30 in ES cell-fate specification by regulation of H3K4 methylation within bivalent domains. Cell 144, 513-525 (2011).

30. Metzger, E. et al. LSD1 demethylates repressive histone marks to promote androgen-receptor-dependent transcription. Nature 437, 436-439 (2005)

31. Shi, X. et al. ING2 PHD domain links histone H3 lysine 4 methylation to active gene repression. Nature 442, 96-99 (2006).

32. Yokoyama, A., Takezawa, S., Schule, R., Kitagawa, H. \& Kato, S. Transrepressive function of TLX requires the histone demethylase LSD1. Mol. Cell Biol. 28, 3995-4003 (2008).

33. Li, W. et al. Nuclear receptor TLX regulates cell cycle progression in neural stem cells of the developing brain. Mol. Endocrinol. 22, 56-64 (2008).

34. Wang, L., Rajan, H., Pitman, J. L., McKeown, M. \& Tsai, C. C. Histone deacetylase-associating Atrophin proteins are nuclear receptor corepressors. Genes Dev. 20, 525-530 (2006).

35. Louis, S. A. \& Reynolds, B. A. Generation and differentiation of neurospheres from murine embryonic day 14 central nervous system tissue. Methods $\mathrm{Mol}$. Biol. 290, 265-280 (2005)

36. Yang, T. et al. POSH localizes activated Rac1 to control the formation of cytoplasmic dilation of the leading process and neuronal migration. Cell Rep. 2, 640-651 (2012).

37. Xu, Z., Kukekov, N. V. \& Greene, L. A. POSH acts as a scaffold for a multiprotein complex that mediates JNK activation in apoptosis. $E M B O J .22$, 252-261 (2003).

38. Sun, Y. et al. The suppression of CRMP2 expression by bone morphogenetic protein (BMP)-SMAD gradient signaling controls multiple stages of neuronal development. J. Biol. Chem. 285, 39039-39050 (2010).
39. Schmittgen, T. D. \& Livak, K. J. Analyzing real-time PCR data by the comparative C(T) method. Nat. Protoc. 3, 1101-1108 (2008).

\section{Acknowledgements}

We thank Drs J. Wang, Z. Qiu, W. Zou, Z. Yuan and X.J. Li for their constructive advices and providing plasmids; Dr M Rosenfeld for generously providing of the Lsd1 f/f mice; Drs X. Cao and F. Sun for generously providing antibodies; Miss J. Pang for analysing the ChIP-seq results. This work was supported by grants from the Chinese Academy of Sciences (XDA01010105/KSCX2-EW-R-11), the MOST (China) (2014CB942801/ 2012YQ03026006/2012CB517904/2013DFA31990) and the NSF (China) (31430037/ $31271156 / 31270826 / 31471132)$.

\section{Author contributions}

F.Z. designed and performed most of the experiments. D.X. performed some of the IUE and immunostaining analysis. L.Y. helped with some western blot analysis. Y.S. and Z.X. were involved in study design. All authors discussed the results and commented on the manuscript.

\section{Additional information}

Supplementary Information accompanies this paper at http://www.nature.com/ naturecommunications

Competing financial interests: The authors declare no competing financial interests.

Reprints and permission information is available online at http://npg.nature.com/ reprintsandpermissions/

How to cite this article: Zhang, F. et al. Epigenetic regulation of Atrophin1 by lysinespecific demethylase 1 is required for cortical progenitor maintenance. Nat. Commun. 5:5815 doi: $10.1038 /$ ncomms6815 (2014).

\section{(c) (i)}

This work is licensed under a Creative Commons Attribution 4.0 International License. The images or other third party material in this article are included in the article's Creative Commons license, unless indicated otherwise in the credit line; if the material is not included under the Creative Commons license, users will need to obtain permission from the license holder to reproduce the material To view a copy of this license, visit http://creativecommons.org/licenses/by/4.0/ 